\title{
A New Accelerating Creep Constitutive Model and its Parameters Sensitivity Analysis
}

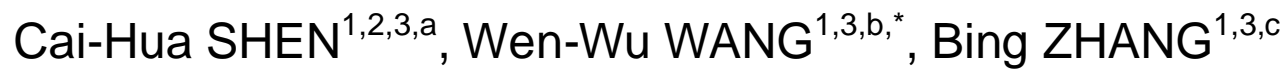 \\ ${ }^{1}$ College of Civil and Transportation Engineering, Hohai University, Nanjing, Jiangsu 210098, China \\ ${ }^{2}$ State Key Laboratory of Geomechanics and Deep Underground Engineering, China University of \\ Mining and Technology, Xuzhou, Jiangsu 221008, China \\ ${ }^{3}$ Key Laboratory of Ministry of Education for Geomechanics and Embankment Engineering, Hohai \\ University, Nanjing, Jiangsu 210098, China \\ asshencaihua@163.com, bwang2012ww@163.com, ‘524548991@qq.com \\ ${ }^{*}$ Corresponding author
}

Keywords: Perzyna Visco-plasticity, Internal Energy Theory, Creep, Parameters Sensitivity.

\begin{abstract}
In this study, Perzyna visco-plasticity theory and Nishihara component model were combined to establish a universal creep constitutive model for describing the entire process of creep, including transient creep, steady-state creep and accelerating creep. Based on the internal energy theory, the constitutive model defined the critical internal energy density value of accelerating creep to describe the mutation characteristics of materials' creep behavior and predict the time of accelerating creep effectively. Overall, the numerical simulation results agreed with the experimental data. Furthermore, according to the sensitivity analysis of parameters, such as flow rule, cohesive force, internal friction angel and Poisson's ratio, and study the constitutive equation change rule with the change of major parameters to further verify the rationality of model. Research shows that, the flow rule and internal friction angle have influence on the time of accelerating creep, and the influence law is close to a parabola form. But the influence law shows a straight line form for the cohesive force and Poisson's ratio. The flow rule, cohesive force and internal friction angle affect steady-state creep strain rate, and the influence law is close to a straight line form. However, Poisson's ratio has no effect on it. The parameters sensitivity of steady-state creep strain rate from small to large in the order is Poisson's ratio, flow rule, cohesive force and internal friction angle. But the sequence turns into Poisson's ratio, cohesive force, flow rule and internal friction angle for the time of accelerating creep.
\end{abstract}

\section{Introduction}

Rock rheology refers to the time-dependent properties of flow, deformation and failure with respect to varying temperature and/or external loading conditions within geological formations; as such, it comprises examination of phenomena such as creep, relaxation, residual elasticity and aging strength, among which creep [1] is defined as 'continuously increasing strain with respect to time under constant external stresses'. Due to the direct engineering implications of rock creep, it now represents a significant research area within rock rheology $[2,3]$.

Because the accelerating creep phase plays a crucial role in stability in engineering applications, it has attracted significant attention from researchers. Vëiìalov [4] considered the nonlinear relation between stress and strain rate, found that the viscosity coefficient of the viscous component varied in the Bingham model, and developed a modified Bingham model. Zhao et al. [5] used the instantaneous elasticity, Hooke body, viscoelasto-plastic Schiffman body, and viscoplastic improved Bingham body to establish a new nonlinear elastovisco-plastic rheological model of rock in series. This model considered the nonlinear characteristics of decay creep and accelerating creep stages and was able to reasonably simulate the accelerating rock creep. Chen et al. [6] proposed two nonlinear components, plastic cranny body and creep body, and established a new nonlinear multiple rheological model. Cao et al. [7] proposed a nonlinear Nishihara model based on an analysis of complete stress-strain curves and a creep process curve. In this model, the viscosity 
coefficient of the viscous component initially increased but subsequently decreased, which could reflect the accelerating rock creep stage. In general, current theoretical studies of rock rheology have mainly focused on component theory, i.e., developing serial and parallel models of basic components (Hooke body, St. Venant body, and Newton body) [8] based on elasticity, plasticity and viscosity. Widely used models include the Kelvin, Burgers, Nishihara, Sterpi and Gioda models [9, 10]. Among these models, significant attention has been given to the Nishihara model [1] (Fig. 1) due to its simplicity and ability to provide a clear, comprehensive reflection of the elastic-viscoelastic-viscoplastic features of rock rheology.

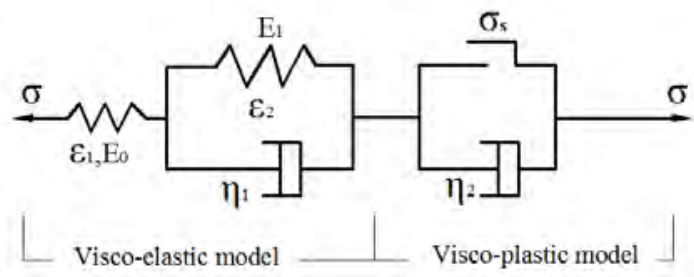

Fig. 1 Structure of the Nishihara Creep Model

The classical Nishihara model contains the Hooke body $(\mathrm{H})$, the viscoelastic body $(\mathrm{N} / \mathrm{H})$ and the viscoplastic body $\left(\mathrm{N} / \mathrm{St}\right.$. V). In Fig. $1, E_{0}$ is the elastic modulus, $E_{1}$ is the viscoelastic modulus, and $\eta_{1}$ and $\eta_{2}$ are the viscosity coefficients of the dashpot. $\sigma$ is total stress, $\mathcal{E}$ is the total strain, $\sigma_{s}$ is the long term strength, and $t_{0}$ is the initial yield time. The one-dimensional Nishihara constitutive equations are shown below in Eq.(1).

$$
\varepsilon(t)= \begin{cases}\frac{\sigma}{E_{0}}+\frac{\sigma}{E_{1}}\left\lfloor 1-\exp \left(-\frac{E_{1}}{\eta_{1}} t\right)\right] & \sigma<\sigma_{s} \\ \frac{\sigma}{E_{0}}+\frac{\sigma}{E_{1}}\left[1-\exp \left(-\frac{E_{1}}{\eta_{1}} t\right)\right]+\eta_{2} \cdot\left(\sigma-\sigma_{s}\right) \cdot\left(t-t_{0}\right) & \sigma \geq \sigma_{s}\end{cases}
$$

The classical Nishihara model is not capable of simulating the rupture of accelerating creep. Therefore, many researchers have modified the Nishihara model to improve the results found when using the model for the accelerating creep state. Li et al. [11] proposed a series of multiple Poynting-Thomson models to simulate nonlinear creep states by using one-dimensional component models of $\mathrm{H}-(\mathrm{N} \|$ St.V), $-\mathrm{N}-(\mathrm{N} \|$ St.V) and $-\mathrm{N}-(\mathrm{N} \|$ St.V)-H-(H\|N). Jiang et al. [12] applied a method with an increasing nonlinear dashpot to simulate accelerating creep processes and added a strain-triggered inertial element in series. In this case, when the strain is larger than the control strain $\varepsilon_{a}$, accelerating creep occurs. Using fractional calculus operator theory and the classical Nishihara model, Zhou et al. [13] proposed a salt rock creep constitutive model that was based on fractional derivatives. These authors used salt rock creep experimental data to validate the model and found good agreement with the fitting results.

In recent years, great progress achieved for the nonlinear component combined model. Because of the model bears the advantages of classical component combined model. However, the model had been presented is only initially formed rock nonlinear component creep model theory, which is imperfect. Currently, the model analysis is mainly aimed at mechanical behavior of the moment after viscoplastic, and the main idea or method is representation research under the condition of fitting experimental data. The simple use of component theory, it is difficult to comprehensively elaborate the occurrence mechanism of creep from mechanical mechanism. Therefore, this paper considered creep mechanism and macro-mechanics behavior as important factors for exploring the mechanism of material failure process. Meanwhile, a nonlinear creep constitutive model was established by combining the Perzyna visco-plasticity theory and the Nishihara component model based on the internal energy theory. Through detailed analysis of the regular pattern of the main physical and mechanics parameters influence simulation of rock creep process, not only further 
validated the rationality of this constitutive model, but also provided reference for better application of constitutive model.

\section{Accelerating Creep Constitutive Equation based on Internal Energy Theory}

Perzyna visco-plasticity theory [14] can be used to accurately simulate the nonlinear viscoplastic mechanical features of the simulated materials, where the viscoplastic strain rate constitutive equation is defined as follows:

$$
\left\{\dot{\varepsilon}^{v p}\right\}=\eta^{\prime} \cdot\langle\varphi(F)\rangle \cdot\{m\}
$$

Here, $\eta^{\prime}$ is the viscoplastic coefficient, $\{m\}$ is the viscoplastic flow direction, and $\phi(F)$ is an arbitrary function of the yield function $F$, which is expressed as

$$
\varphi(F)=\left(\frac{F}{F_{0}}\right)^{n}
$$

When the creep process does not change the stress state, the plastic flow (creep) in elastoplastic theory can be considered as related to the internal energy increment of the material. Therefore,

$$
F=\beta \cdot \sigma_{s i} \cdot \varepsilon_{s i}\left(\sigma_{s i}, t\right)
$$

Where $\beta$ is the equivalent coefficient of the stress state (which is associated with the second principal stress and which is approximately 1 in the single-axis or plane triaxial test), $\sigma_{s i}$ is any stress greater than $\sigma_{s}, \varepsilon_{s i}\left(\sigma_{s i}, t\right)$ is the creep strain (which is a complex function in progressive failure analyses). When $\sigma_{s i}$ is constant, $\varepsilon_{s i}\left(\sigma_{s i}, t\right)$ can be simplified as a function of time $\varepsilon_{s i}(t)$. Based on an infinite polynomial approximation of the creep process curve

$$
\varepsilon_{s i}(t)=\varepsilon_{s l} \cdot f(t)
$$

Where $f(t)=a_{0}+a_{1}\left(t-\tau_{0}\right)+\ldots+a_{i}\left(t-\tau_{0}\right)^{i}+\ldots+a_{k}\left(t-\tau_{0}\right)^{k}, \quad k=0 \sim N ; \quad a_{i} \quad$ is a constant coefficient; and $\tau_{0}$ is the occurrence time of the non-zero steady-state creep.

$$
F_{0}=\beta \cdot \sigma_{s} \cdot \varepsilon_{s l}
$$

Here, $\sigma_{s}$ is the long-term strength value, namely, the minimum equivalent stress of the strain state occurring for the non-zero steady-state creep. In addition, $\varepsilon_{s l}$ is the strain value at the beginning time of the non-zero steady-state creep.

Therefore, combining the viscoplastic Eq. (2) and Nishihara models improved the Nishihara model, and the constitutive equations of the viscoelastic and viscoplastic stages can be written as follows:

$$
\varepsilon(t)= \begin{cases}\frac{\sigma}{E_{0}}+\frac{\sigma}{E_{1}}\left\lfloor 1-\exp \left(-\frac{E_{1}}{\eta_{1}} t\right)\right] & F \leq F \\ \frac{\sigma}{E_{0}}+\frac{\sigma}{E_{1}}\left[1-\exp \left(-\frac{E_{1}}{\eta_{1}} t\right)\right]+\eta_{2} \cdot\left\langle\frac{\sigma \cdot f(t)}{\sigma_{0}}\right\rangle^{n} \cdot t & F>F_{0}\end{cases}
$$

To simplify the calculation, it is desirable that $n=1$. When $k=1, f(t)=a_{0}+a_{1} \cdot\left(t-\tau_{0}\right)$, and 
the creep unified constitutive equation degenerates into a method with an increasing nonlinear dashpot to simulate the accelerating creep process [12].

According to the triaxial test, when a material exhibits a non-zero steady-state creep, it will achieve accelerating creep as the creep strain energy increases. Here, it was assumed that the materials would enter the accelerating creep stage when the creep strain energy increment reaches the critical value. From the internal energy perspective (e.g., defined as the accelerating creep critical strain energy value $\Gamma_{a}^{*}$ ), it can be concluded that the critical value is a constant that does not change when the stress state changes. Therefore, the critical value can be determined from one accelerating creep test curve. In addition, The internal energy change can be used to distinguish the behavior of the entire rock creep process.

When $F \leq F_{0}$, the materials are in the viscoelastic stage; when $F_{0}<F<F_{0 l}$, the materials enter the steady-creep stage; and when $F \geq F_{0 l}$, the materials reach the accelerating creep stage.

Clearly, $F \leq F_{0}$ is equivalent to $\sigma \leq \sigma_{s}, \quad F_{0}<F<F_{0 l}$ is equivalent to $\sigma>\sigma_{s}$ and $\sigma \cdot \varepsilon_{s}<\sigma_{s} \cdot \varepsilon_{s}^{a p}$, and $F \geq F_{0 l}$ is equivalent to $\sigma \cdot \varepsilon_{s} \geq \sigma_{s} \cdot \varepsilon_{s}^{a p}=\Gamma_{a}^{*}$.

Here, $\varepsilon_{s}$ is the creep strain (e.g., total strain minus instantaneous strain), and $\varepsilon_{s}^{a p}$ is the creep strain at the beginning of the accelerating creep stage in the ideal long-term strength state.

For the stress state $\sigma_{i}>\sigma_{s}$, the creep strain at the beginning of accelerating creep becomes $\varepsilon_{s i}^{a p}$. According to the critical energy value $\Gamma_{a}^{*}$ of accelerating creep, the definition becomes

$$
\Gamma_{a}^{*}=\sigma_{i} \cdot \varepsilon_{s i}^{a p}=\sigma_{s} \cdot \varepsilon_{s}^{a p}
$$

According to the real situation, the increasing stress state influences the speed of the accelerating creep. Therefore, the selection of $k$ in $f(t)$ (a nonlinear characteristic parameter of creep reflecting the magnitude of creep acceleration) should be related to the yield stress ratio $\frac{\sigma}{\sigma_{0}}$ [15]. To simplify the calculation,

$$
\left(t-\tau_{0}\right) \cdot f\left(t-\tau_{0}\right)=a_{k}\left(t-\tau_{0}\right)^{\left(\frac{\sigma}{\sigma_{0}}\right)} \cdot t
$$

Therefore, a simplified, practical creep constitutive model that describes accelerating creep processes was obtained based on strain energy theory.

$$
\begin{aligned}
& \varepsilon(t)=\frac{\sigma}{E_{0}}+\frac{\sigma}{E_{1}}\left\lfloor 1-\exp \left(-\frac{E_{1}}{\eta_{1}} t\right)\right] \quad \sigma \leq \sigma_{s} \\
& \varepsilon(t)=\frac{\sigma}{E_{0}}+\frac{\sigma}{E_{1}}\left[1-\exp \left(-\frac{E_{1}}{\eta_{1}} t\right)\right]+\eta_{2}^{\prime} \cdot\left\langle\frac{\sigma}{\sigma_{0}}\right\rangle \cdot t \quad \sigma>\sigma_{s} \text { and } \sigma \cdot \varepsilon_{s}<\sigma_{s} \cdot \varepsilon_{s}^{a p}=\Gamma_{a}^{*} \\
& \varepsilon(t)=\frac{\sigma}{E_{0}}+\frac{\sigma}{E_{1}}\left[1-\exp \left(-\frac{E_{1}}{\eta_{1}} t\right)\right]+\eta_{2}^{\prime} \cdot\left\langle\frac{\sigma}{\sigma_{0}}\right\rangle \cdot \tau_{0}+\eta_{2}^{\prime} \cdot \eta_{3}^{\prime} \cdot\left\langle\frac{\sigma}{\sigma_{0}}\right\rangle \cdot\left(t-\tau_{0}\right)^{\frac{\sigma}{\sigma_{0}}} \cdot t \quad \sigma \cdot \varepsilon_{s} \geq \sigma_{s} \cdot \varepsilon_{s}^{a p}=\Gamma_{a}^{*}
\end{aligned}
$$

The one-dimensional constitutive equations were rewritten in three-dimensional matrix format as follows:

$$
\{\varepsilon(t)\}=\left(\frac{1}{E_{0}}+\frac{1}{E_{1}^{\prime}}\right)[A]\{\sigma\} \quad \bar{\sigma} \leq \bar{\sigma}_{s}
$$




$$
\begin{aligned}
& \{\varepsilon(t)\}=\left(\frac{1}{E_{0}}+\frac{1}{E_{1}^{\prime}}\right)[A]\{\sigma\}+\eta_{2}^{\prime}\left\langle\frac{F}{\bar{\sigma}_{0}}\right)\{m\} \cdot t \quad \bar{\sigma}>\bar{\sigma}_{s} \text { and } \bar{\sigma} \cdot \bar{\varepsilon}_{s}<\bar{\sigma}_{s} \cdot \bar{\varepsilon}_{s}^{a p}=\Gamma_{a}^{*} \\
& \{\varepsilon(t)\}=\left(\frac{1}{E_{0}}+\frac{1}{E_{1}^{\prime}}\right)[A]\{\sigma\}+\eta_{2}^{\prime}\left(\frac{F}{\bar{\sigma}_{0}}\right)\{m\} \cdot \tau_{0}+\eta_{2}^{\prime} \cdot \eta_{3}^{\prime} \cdot\left(t-\tau_{0}\right)^{\left(\frac{F}{\bar{\sigma}_{0}}\right)}\left(\frac{F}{\bar{\sigma}_{0}}\right)\{m\} \cdot t \quad \bar{\sigma} \cdot \bar{\varepsilon}_{s} \geq \bar{\sigma}_{s} \cdot \bar{\varepsilon}_{s}^{a p}=\Gamma_{a}^{*}
\end{aligned}
$$

where the variables with over-lines represent the yield strength calculation values under three-dimensional conditions.

$$
\begin{aligned}
E_{1}^{\prime} & =\frac{E_{1}}{1-\exp \left(-\frac{E_{1}}{\eta_{1}} t\right)} \\
{[A] } & =\left[\begin{array}{cccccc}
1 & -\mu & -\mu & 0 & 0 & 0 \\
-\mu & 1 & -\mu & 0 & 0 & 0 \\
-\mu & -\mu & 1 & 0 & 0 & 0 \\
0 & 0 & 0 & 2(1+\mu) & 0 & 0 \\
0 & 0 & 0 & 0 & 2(1+\mu) & 0 \\
0 & 0 & 0 & 0 & 0 & 2(1+\mu)
\end{array}\right] \\
\{m\} & =\frac{\partial F}{\partial \sigma}
\end{aligned}
$$

\section{Parameters Sensitivity Analysis of Accelerating Creep Constitutive Model Based on the D-P Yield Criterion}

Here assumption is made that yield criterion in Eq.(13), (14),(15) adopts the D-P yield criterion: $F=\alpha I_{1}+\sqrt{J_{2}}, \quad I_{1}=\sigma_{x}+\sigma_{y}+\sigma_{z}$.

Here, $\alpha=\frac{2 \sin \theta}{\sqrt{3}(3-\sin \theta)}, \theta$ is the internal friction angle. Therefore, the accelerating creep constitutive model based on D-P yield criterion can be established.

\section{Determine the Critical Internal Energy Density Value of Accelerating Creep by Experimental Curves}

The triaxial creep tests specimens are sandstone specimens. The axial pressure was $61 \mathrm{Mpa}$, and the confining pressure was $6.0 \mathrm{Mpa}$. Using the least squares approach, the parameters of the proposed constitutive model can be determined by fitting Eqs. (13), (14), and (15). By applying the Drucker-Prager yield criterion and the associated flow rule, a three-dimensional constitutive model can be established. The parameters that were obtained by fitting the experimental data are shown in table 1. The data from the triaxial test and the fitting curve of modified Nishihara model are shown in Fig. 2. The measured accelerating creep strain energy density was $0.150 \mathrm{~J} / \mathrm{m}^{3}$ at the time of accelerating creep $\left(\tau_{2}=36.2 \mathrm{hr}\right)$.

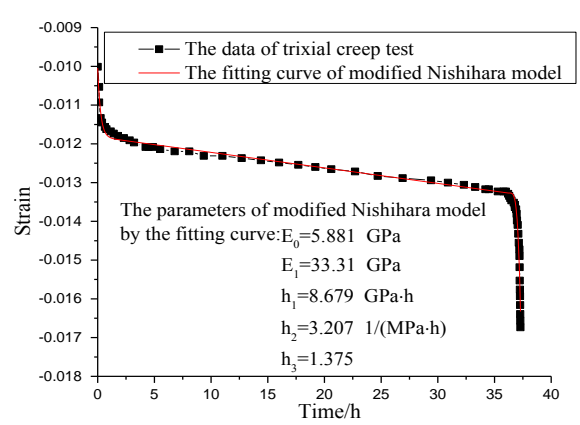

Fig. 2 the Data from the Triaxial Test and the Fitting Curve of the Modified Nishihara Model 
Tab. 1 Parameters of the Triaxial Creep Tests and the Parameters of Constitutive Model

\begin{tabular}{|c|c|c|c|c|c|c|c|c|}
\hline \multirow{2}{*}{ Cohesion } & \multirow{2}{*}{$\begin{array}{l}\text { Fiction } \\
\text { angle }\end{array}$} & \multirow{2}{*}{\multicolumn{2}{|c|}{$\begin{array}{l}\text { Poisson's } \\
\text { ratio }\end{array}$}} & \multicolumn{5}{|c|}{$\begin{array}{c}\text { Calculated parameters of triaxial creep } \\
\text { tests }\end{array}$} \\
\hline & & & & $b_{0}$ & $b_{1}$ & & $b_{2}$ & $\Gamma_{a}^{*}$ \\
\hline$c(M P a)$ & $\theta\left({ }^{\circ}\right)$ & & $M P a$ & 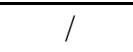 & & $J / m^{3}$ \\
\hline 1.48 & 42 & \multicolumn{2}{|c|}{0.16} & -59.08 & 4.6225 & \multicolumn{2}{|c|}{-0.2699} & 0.150 \\
\hline \multicolumn{9}{|c|}{ Parameters of modified Nishihara model by the fitting } \\
\hline $\begin{array}{l}\text { Elasticity } \\
\text { modulus } \\
E_{0}(G P a)\end{array}$ & \multicolumn{2}{|c|}{$\begin{array}{l}\text { Elasticity } \\
\text { modulus } \\
E_{1}(G P a)\end{array}$} & \multicolumn{2}{|c|}{$\begin{array}{c}\text { Viscosity } \\
\text { coefficient } \\
\eta_{1}^{\prime} \\
(G P a \cdot h)\end{array}$} & \multicolumn{2}{|c|}{$\begin{array}{c}\text { Viscosity } \\
\text { coefficient } \\
\eta_{2}^{\prime} \\
\left((G P a \cdot h)^{-1}\right)\end{array}$} & \multicolumn{2}{|c|}{$\begin{array}{c}\text { Equivalent } \\
\text { coefficient } \\
\eta_{3}^{\prime}\end{array}$} \\
\hline 5.881 & \multicolumn{2}{|c|}{33.31} & \multicolumn{2}{|c|}{8.679} & 3.207 & & \multicolumn{2}{|c|}{1.375} \\
\hline
\end{tabular}

\section{Parameters Sensitivity Analysis of the Constitutive Equation}
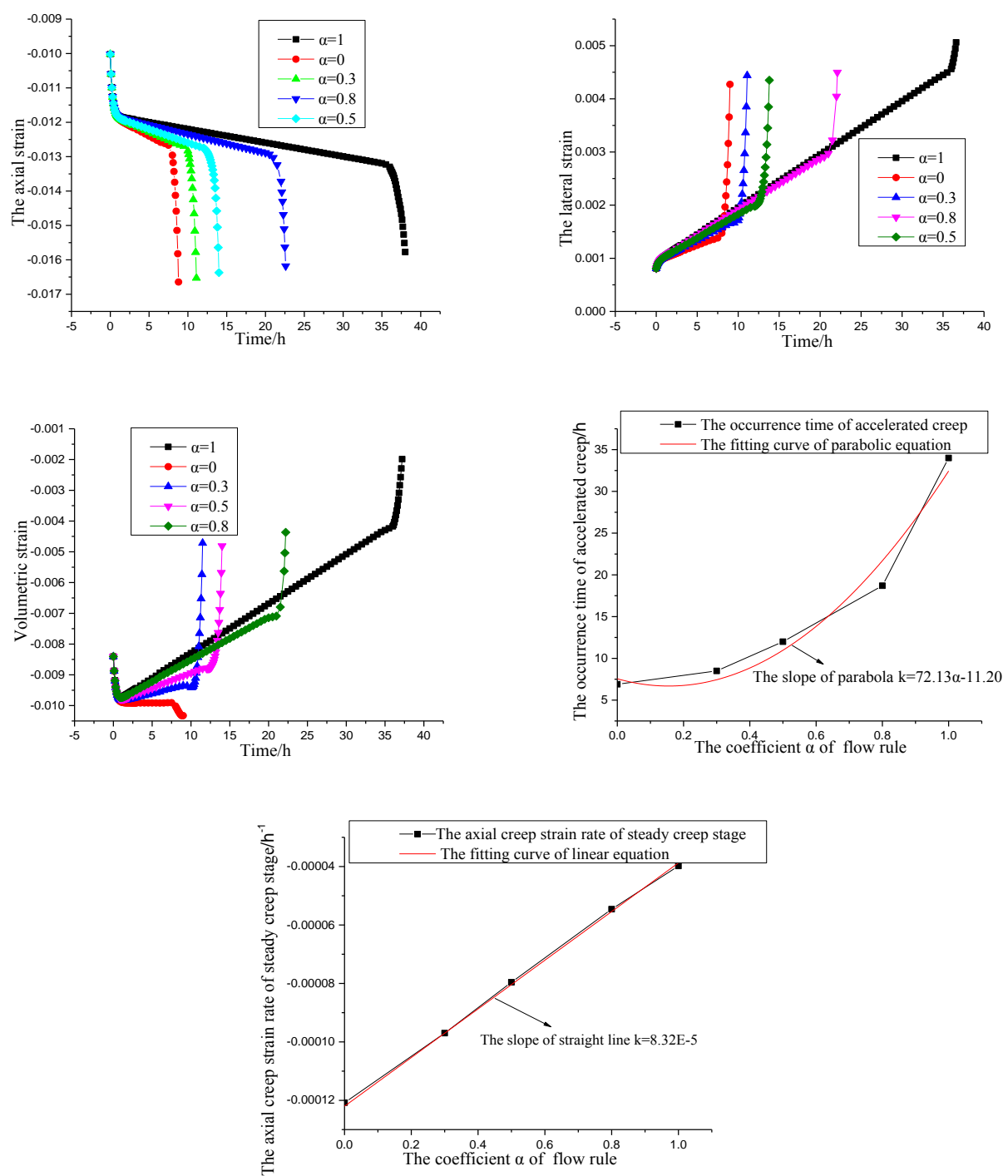

Fig. 3 the Triaxial Creep Process and Parameters Sensitivity in different Flow Rules

To validate the constitutive model better, parameters sensitivity analysis was adopted to research the constitutive equation change law with main parameters change and verify whether the constitutive equation is reasonable.

Therefore, according to the sensitivity analysis of parameters, such as flow rule, cohesive force, 
internal friction angel and Poisson's ratio, and study the constitutive equation change rule with the change of major parameters to further verify the rationality of model.

Analysis of the Effects of Flow Rule Variations on the Prediction of Accelerating Creep. Fig. 3 are the simulated triaxial creep results using constitutive model in four different flow rules of $\alpha=0, \alpha=0.3, \alpha=0.5$ and $\alpha=0.8$ (non-associated flow rule) with an axial pressure of 61 $\mathrm{MPa}$ and with confining pressures of $6.0 \mathrm{MPa}$, compared with the simulated results in the flow rule of $\alpha=1$ (associated flow rule). Analysis of the effects of flow rule variations is on the prediction of the triaxial creep process.

Analysis of the Effects of Cohesive Force Variations on the Prediction of Accelerating Creep. The cohesive force and internal friction angle of rock are very difficult to determine experimentally. Therefore, one research challenge is the selection of parameters for the constitutive model. Fig. 4 shows the research results that analysis of the effects of cohesive force variations on the prediction of the triaxial creep process in the flow rule of $\alpha=1$.
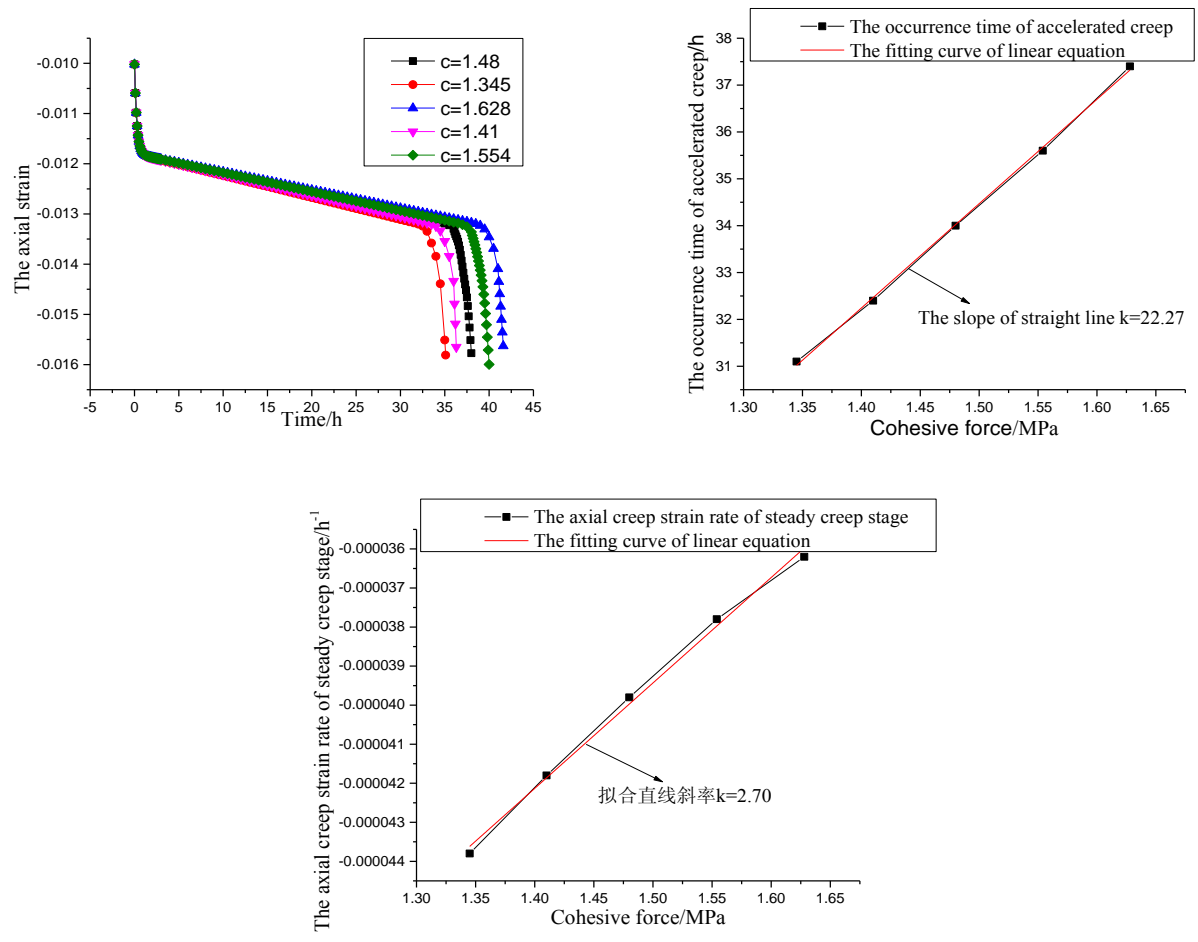

Fig. 4 the Triaxial Creep Process and Parameters Sensitivity in Different Cohesive Forces

Analysis of the Effects of Internal Friction Angel Variations on the Prediction of Accelerating Creep. Fig. 5 shows the research results that analysis of the effects of internal friction variations on the prediction of the triaxial creep process in the flow rule of $\alpha=1$.
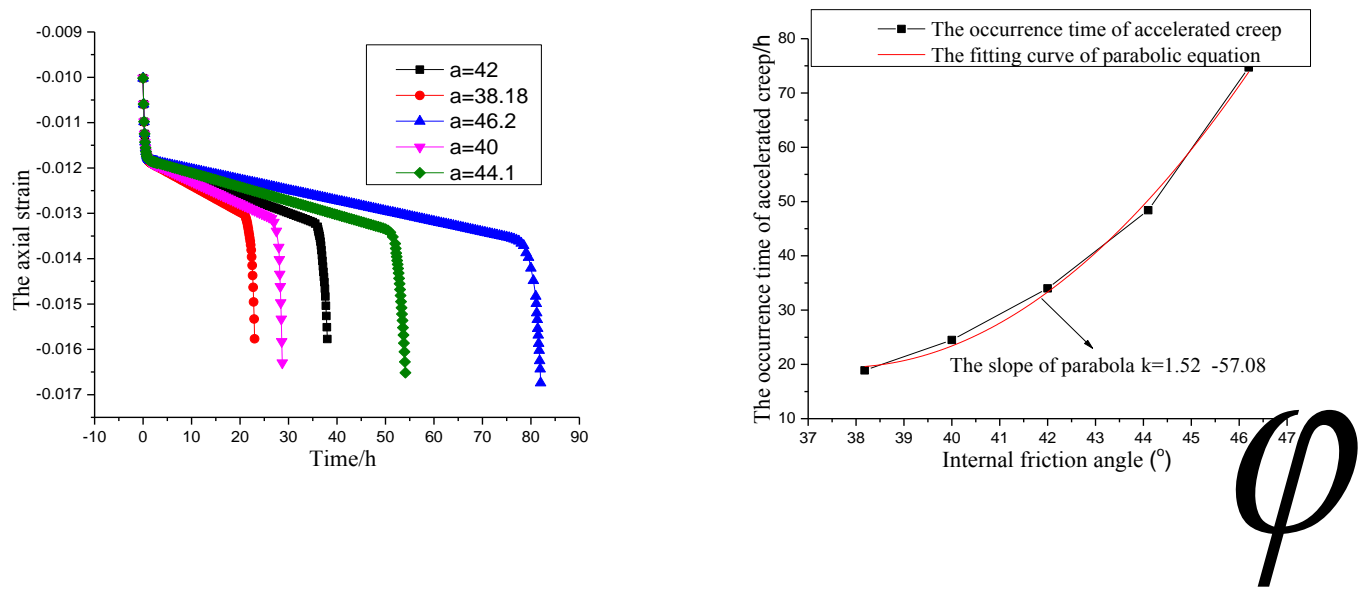


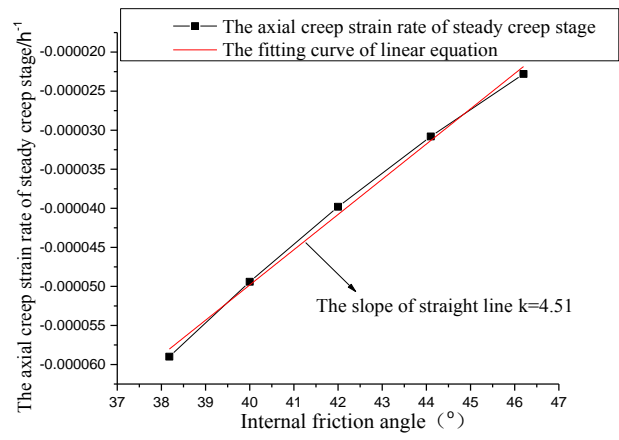

Fig. 5 the Triaxial Creep Process and Parameters Sensitivity in Different Internal Friction Angle

Analysis of the Effects of Poisson Ratio Variations on the Prediction of Accelerating Creep. Fig. 6 shows the research results that analysis of the effects of five different Poisson ratio ( $\mu=0.16, \mu=0.18, \mu=0.2, \mu=0.25$ and $\mu=0.3$ ) on the prediction of the triaxial creep process in the flow rule of $\alpha=1$.
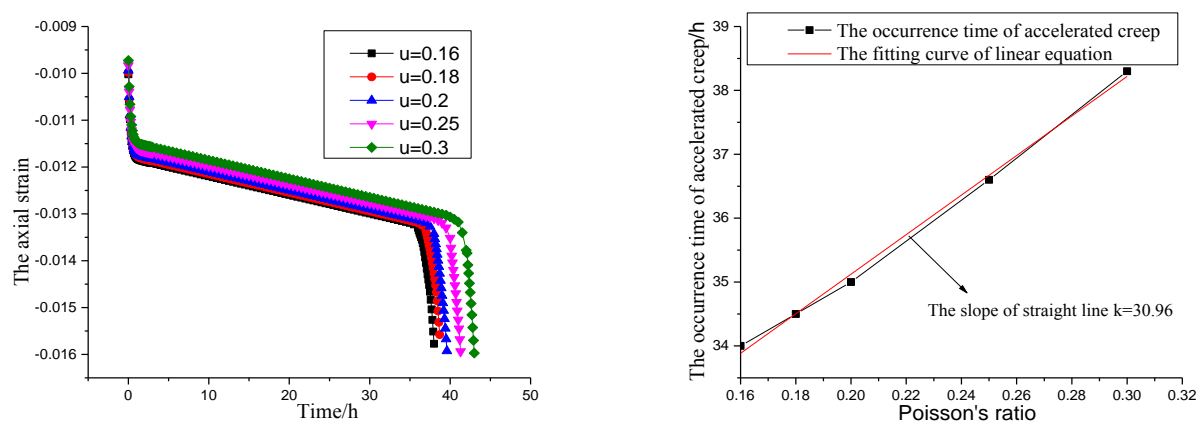

Fig. 6 the Triaxial Creep Process and Parameters Sensitivity in Different Poisson's Ratio

Fig. $3 \sim 6$ shows that it has a great influence on the prediction of the accelerating creep time and the steady-state creep strain rata. In order to study the effects of different parameters in the constitutive model well, this paper would analyze parameters sensitivity. Here, the flow rule $\alpha$ predicts sensitivity coefficient of the time of accelerating creep as follow:

$$
\gamma_{\alpha}=\left(\Delta t \cdot \alpha_{0}\right) /\left(t_{0} \cdot \Delta \alpha\right)
$$

The flow rule $\alpha$ predicts sensitivity coefficient of the steady-state creep strain rata.

$$
v_{\alpha}=\left(\Delta \kappa \cdot \alpha_{0}\right) /\left(\kappa_{0} \cdot \Delta \alpha\right)
$$

Here, $\gamma_{\alpha}$ is the sensitivity coefficient of the time of accelerating creep, $v_{\alpha}$ is the sensitivity coefficient of the steady-state creep strain rata, $\Delta t$ is the different value of the occurrence time of accelerating creep, $\Delta \alpha$ is the different value of the flow rule coefficient, $\Delta \kappa$ is the different value of the steady-state creep strain rata, $\alpha_{0}$ is one of the flow rule, $t_{0}$ is the time of accelerating creep, $\kappa_{0}$ is the steady-state creep strain rata.

The cohesive force, internal friction angel and Poisson's ratio predict sensitivity coefficient of the time of accelerating creep and the steady-state creep strain rata as follows:

$$
\gamma_{c}=\left(\Delta t \cdot c_{0}\right) /\left(t_{0} \cdot \Delta c\right), \quad v_{c}=\left(\Delta \kappa \cdot c_{0}\right) /\left(\kappa_{0} \cdot \Delta c\right)
$$




$$
\begin{aligned}
& \gamma_{\varphi}=\left(\Delta t \cdot \varphi_{0}\right) /\left(t_{0} \cdot \Delta \varphi\right), \quad v_{\varphi}=\left(\Delta \kappa \cdot \varphi_{0}\right) /\left(\kappa_{0} \cdot \Delta \varphi\right) \\
& \gamma_{\mu}=\left(\Delta t \cdot \mu_{0}\right) /\left(t_{0} \cdot \Delta \mu\right), \quad v_{\alpha}=\left(\Delta \kappa \cdot \alpha_{0}\right) /\left(\kappa_{0} \cdot \Delta \alpha\right)
\end{aligned}
$$

The sensitivity coefficients of the effects of main parameters variations on the simulation of rock creep processes are shown in table 2 .

\begin{tabular}{|c|c|c|c|c|c|c|c|c|c|}
\hline \multirow{3}{*}{ main parameters } & \multicolumn{3}{|c|}{ Flow rule coefficients } & \multicolumn{2}{|c|}{$\begin{array}{l}\text { cohesive } \\
\text { force }\end{array}$} & \multicolumn{2}{|c|}{$\begin{array}{c}\text { internal friction } \\
\text { angle }\end{array}$} & \multicolumn{2}{|c|}{$\begin{array}{c}\text { Poisson's } \\
\text { ratio } \\
\end{array}$} \\
\hline & \multicolumn{2}{|c|}{$\gamma_{\alpha}$} & \multirow{2}{*}{$v_{\alpha}$} & \multirow{2}{*}{$\gamma_{c}$} & \multirow{2}{*}{$v_{c}$} & \multirow{2}{*}{$\gamma_{\varphi}$} & \multirow{2}{*}{$v_{\varphi}$} & \multirow{2}{*}{$\gamma_{\mu}$} & \multirow{2}{*}{$v_{\mu}$} \\
\hline & $\alpha=0 \sim 0.8$ & $\alpha=0.8 \sim 1$ & & & & & & & \\
\hline $\begin{array}{c}\text { sensitivity } \\
\text { coefficients } \\
\text { (average value) }\end{array}$ & 0.641 & 3.273 & 0.269 & 0.992 & $\begin{array}{c}0.97 \\
5\end{array}$ & 8.463 & 4.217 & $\begin{array}{c}0.18 \\
2\end{array}$ & 0 \\
\hline
\end{tabular}

Tab. 2 Analysis of the Parameter Sensitivity

Research shows that, the flow rule and internal friction angle have influence on the time of accelerating creep, and the influence law curve is close to a parabola. But the influence law shows a straight line form for cohesive force and Poisson's ratio. The flow rule, cohesive force and internal friction angle affect steady-state creep ratio, and the influence law curve is close to a straight line form. However, Poisson's ratio has no effect on it.The parameters sensitivity of steady-state creep ratio from small to large in the order is Poisson's ratio, flow rule, cohesive force and internal friction angle. But the sequence turns into Poisson's ratio, cohesive force, flow rule and internal friction angle for the occurrence time of accelerating creep.

\section{Conclusion}

1. This paper developed a modified Nishihara model based on Perzyna viscoplastic theory, which combines nonlinear viscoplastic theory with a component method. This model is easy to understand and reflects the nonlinear process of creep, providing insights for research into simulating the rock creep process.

2. Based on internal energy theory, this paper used the critical internal energy density value of accelerating creep to describe the properties of materials undergoing sudden changes in creep mechanics. According to the sensitivity analysis of parameters, such as flow rule, cohesive force, internal friction angel and Poisson's ratio, and study the constitutive equation change rule with the change of major parameters to further verify the rationality of model. The proposed method for predicting accelerating creep and analyzing parameters sensitivity in this study was based on the critical internal energy density of accelerating creep and provided new insights into the study of rock creep macro-constitutive models.

\section{Acknowledgement}

The work reported in this paper has been supported by Natural Science Foundation of Jiangsu Province of China (Grant No. BK20141419), and supported by State Key Laboratory for GeoMechanics and Deep Underground Engineering, China University of Mining \& Technology (Grant No. SKLGDUEK1211), and supported by the Fundamental Research Funds for the Central Universities (Grant No. 2014B04914).The authors gratefully acknowledge these supports.

\section{References}

[1]NISHIHARA M: 'Creep of shale and sandy-shale', Geological Magazine 1952, 58(683), 373-377.

[2]Atkinson B K: 'Subcritical crack propagation in rocks: theory, experimental results and 
applications', Journal of Structural Geology 1982, 4(1), 41-56.

[3]Atkinson B K and Meredith P G: 'The theory of subcritical crack growth with applications to minerals and rocks', Fracture mechanics of rock 1987, 2, 111-166.

[4]Vëiìalov S S: 'Rheological fundamentals of soil mechanics', Elsevier (Amsterdam and New York and New York, NY), 1986.

[5]Zhao YL, Cao P, Wen YD, Wang YX and Chai HB: 'Elastovisco-plastic rheological experiment and nonlinear rheological model of rocks', Chinese Journal of Rock Mechanics and Engineering, 2008, 27(3), 477-486.

[6]Chen YJ: 'Study on rheological constitutive model of rock and its intelligent identification', $\mathrm{PhD}$ thesis, Central South University, China, 2003.

[7]Cao SG, Bian J and Li P: 'Rheologic constitutive relationship of rocks and a modified model', Chinese Journal of Rock Mechanics and Engineering 2002, 21 (5), 632 - 634.

[8]De Borst R, Crisfield M A, Remmers J J and Verhoosel C V: 'Time-dependent Material Models', Non-Linear Finite Element Analysis of Solids and Structures, Second Edition, 2012, 281-304.

[9]Sterpi D and Gioda G: 'Visco-plastic behaviour around advancing tunnels in squeezing rock', Rock Mechanics and Rock Engineering, 2009, 42(2), 319-339.

[10]Tang M and Wang Z: 'Experimental study on rheological deformation and stress properties of limestone', Journal of Central South University of Technology, 2008, 15(1), 475-478.

[11]Li HT, Tan YL, Ning JG and Yan WZ: 'Creep model for Zonal disintegration of deep rock mass', In: 'Electric Information and Control Engineering (ICEICE)', 2011 International Conference on, IEEE 2011, 5780-5783.

[12]Jiang QH, Qi YJ, Wang ZJ and Zhou CB: 'An extended Nishihara model for the description of three stages of sandstone creep', Geophysical Journal Internationall, 2013, 193(2), 841-854.

[13]Zhou HW, Wang CP, Han BB and Duan ZQ: 'A creep constitutive model for salt rock based on fractional derivatives', International Journal of Rock Mechanics and Mining Sciences, 2011, 48(1), 116-121.

[14]Zhou HW, Wang CP and Mishnaevsky Jr L: 'A fractional derivative approach to full creep regions in salt rock', Mechanics of Time-Dependent Materials, 2013, 17(3), 413-425.

[15]Rutter E: 'On the creep testing of rocks at constant stress and constant force', In: International Journal of Rock Mechanics and Mining Sciences \& Geomechanics Abstracts. Elsevier, 1972, 191-195. 